\title{
On some extension of optimal control theory
}

\author{
Dmitry Yu. Karamzin a,b ${ }^{\text {, }}$ Valeriano A. de Oliveira ${ }^{a}$, Fernando L. Pereira ${ }^{c}$, Geraldo N. Silva ${ }^{a, *}$ \\ a Instituto de Biociências, Letras e Ciências Exatas, UNESP - Univ. Estadual Paulista, Rua Cristóvão Colombo, N. 2265, \\ CEP 15054-000, São José do Rio Preto - SP, Brazil \\ ${ }^{\mathrm{b}}$ Computing Centre of RAS, Vavilova Street, 40, 119991, Moscow, Russia \\ ${ }^{\text {c } F a c u l d a d e ~ d e ~ E n g e n h a r i a, ~ U n i v e r s i d a d e ~ d o ~ P o r t o, ~ R u a ~ D r . ~ R o b e r t o ~ F r i a s, ~ s / n ~ 4200-465, ~ P o r t o, ~ P o r t u g a l ~}$
}

\section{A R T I C L E I N F O}

\section{Article history:}

Received 21 April 2014

Received in revised form

15 August 2014

Accepted 29 September 2014

Recommended by J. Tsinias

Available online 23 October 2014

\section{Keywords:}

Optimal control

Impulsive controls

Maximum principle

\begin{abstract}
A B S T R A C T
Some problems of Calculus of Variations do not have solutions in the class of classic continuous and smooth arcs. This suggests the need of a relaxation or extension of the problem ensuring the existence of a solution in some enlarged class of arcs. This work aims at the development of an extension for a more general optimal control problem with nonlinear control dynamics in which the control function takes values in some closed, but not necessarily bounded, set. To achieve this goal, we exploit the approach of R.V. Gamkrelidze based on the generalized controls, but related to discontinuous arcs. This leads to the notion of generalized impulsive control. The proposed extension links various approaches on the issue of extension found in the literature.
\end{abstract}

๑ 2014 European Control Association. Published by Elsevier Ltd. All rights reserved.

\section{Introduction}

It is well known that variational calculus problems may not have a smooth or even continuous solution. Nevertheless, discontinuous solutions may still be of interest from the physical point of view. Consider the following famous Euler example

$\begin{array}{ll}\text { Minimize } & \int_{0}^{1} x(t) \sqrt{1+(\dot{x})^{2}} d t, \\ \text { subject to } & x(0)=r_{1}, \quad x(1)=r_{2}, \\ & x(t) \geq 0 .\end{array}$

This is the so-called minimal surface problem. Physically, the solution $x(\cdot)$ is the shape of a soap bubble or a membrane stretched over two parallel disks with radiuses $r_{1}$ and $r_{2}$. The application of the Euler-Lagrange principle leads to a second order differential equation and to a boundary-value problem, which does not have solutions for certain values of $r_{1}, r_{2}$. The physical meaning is as follows: if numbers $r_{1}, r_{2}$ are sufficiently large relatively to the distance between the disks, the membrane exists and the surface of revolution is smooth. But, if we increase the distance between the disks, the soap bubble stretches and, at some point, blows up. At that very moment, the smooth and continuous solution fails to exist. However, it does not mean that a solution $x(\cdot)$ does not

\footnotetext{
* Corresponding author. Tel.: +55 173221 2209; fax: +55 1732212203 .

E-mail addresses: dmitry_karamzin@mail.ru (D.Yu. Karamzin), antunes@ibilce.unesp.br (V.A. de Oliveira),flp@fe.up.pt (F.L. Pereira), gsilva@ibilce.unesp.br (G.N. Silva).
}

exist at all. In this degenerate case, the solution is $x(0)=r_{1}$, $x(1)=r_{2}, x(t)=0, t \in(0,1)$, and, thus, it exhibits discontinuities.

Consider another famous example, the Dido problem.

Maximize $\int_{0}^{1} x(t) d t$

subject to $\quad x(0)=x(1)=0, \quad x(t) \geq 0$,

$\int_{0}^{1} \sqrt{1+(\dot{x})^{2}} d t=l$

Once again, continuous solution fails to exist when the length of the arc $l$ is sufficiently large. Dido problem is a typical example of the so-called isoperimetric problem. The situation in which there is no solution is fairly common in such kind of problems.

An isoperimetric version of the Euler example is the catenary

Minimize $\int_{0}^{1} x(t) \sqrt{1+(\dot{x})^{2}} d t$

subject to $\quad x(0)=r_{1}, \quad x(1)=r_{2}, \quad x(t) \geq 0$,

$$
\int_{0}^{1} \sqrt{1+(\dot{x})^{2}} d t=l
$$

The equation of the catenary curve was derived by Leibniz, Huygens and Johann Bernoulli in 1691. They were the first ones to find out that this curve is a hyperbolic cosine, and not a parabola as it had been thought before. The shape of the soap bubble (1) is, as we see from (3), again a catenary, and Euler was the first one to prove it by using Variational Calculus. Once again, this problem naturally allows for discontinuous solutions. 
Another simple example of an isoperimetric problem is the one of minimizing the norm of a function in $\mathbb{L}_{1}$ over the elements of the unit sphere in $\mathbb{L}_{2}$

Minimize $\int_{0}^{1}|\dot{x}| d t$

subject to $\quad x(0)=0, \quad \int_{0}^{1}|\dot{x}|^{2} d t=1$.

The solution does not exist. Indeed the infimum, here, is zero, but it is not reached due to the integral constraint.

In the framework of his famous program, David Hilbert [18] suggested (20th problem) to extend the Variational Calculus in order to cover and to formalize such degenerate situations when solutions fail to exist, by giving a strict mathematical meaning to non-smooth and to non-classical solutions as a whole. He expressed his confidence that every problem in the calculus of variations has a solution, provided that the term solution is interpreted appropriately. That spawned a number of theories on the extension of classic variational calculus by various authors. For the rich history of this issue, we refer the reader to the article [21], see also the bibliography therein. Here, we only point out important contributions made by H. Lebesgue, L. Tonelli, L. Young, N. Bogolyubov, R. Gamkrelidze, R. Rockafellar, A. Ioffe, V. Tikhomirov, and J. Warga, among others (see references in the above source for an overview).

So, in the realm of this context, the aim of the current research is to give a strict mathematical meaning to discontinuous solutions that may arise in the following optimal control problem

$$
\begin{array}{ll}
\text { Minimize } & \int_{t_{0}}^{t_{1}} g_{0}(x, v, t) d t, \\
\text { subject to } & \dot{x}=g(x, v, t), \quad t \in\left[t_{0}, t_{1}\right], \\
& x\left(t_{0}\right) \in A, \quad x\left(t_{1}\right) \in B, \\
& v(t) \in V \quad \text { a.a. } t \in\left[t_{0}, t_{1}\right], \\
& \varphi(x(t), t) \leq 0 \quad \forall t \in\left[t_{0}, t_{1}\right],
\end{array}
$$

where $g_{0}: \mathbb{R}^{n} \times \mathbb{R}^{k} \times \mathbb{R}^{1} \rightarrow \mathbb{R}^{1}, g: \mathbb{R}^{n} \times \mathbb{R}^{k} \times \mathbb{R}^{1} \rightarrow \mathbb{R}^{n}$, and $\varphi: \mathbb{R}^{n} \times$ $\mathbb{R}^{1} \rightarrow \mathbb{R}^{l}$ are given continuous maps, $A$ and $B$ are given closed subsets of $\mathbb{R}^{n}, V$ is a given closed, not necessarily bounded, subset of $\mathbb{R}^{k}$ and $v$ $(\cdot)$ is a control function. The function $\varphi$ defines the so-called state constraints.

In what follows, we associate the control problem (5) an a priori given scalar function $\omega(\xi): \mathbb{R}_{+} \rightarrow \mathbb{R}_{+}$which is nonnegative, increasing and continuous. The purpose of introducing this function is to characterize the growth of the dynamics at infinity. Simple examples of $\omega(\xi)$ are: $\xi$ (linear growth), $\xi^{2}$ (quadratic growth), $\xi^{p}, e^{\xi}$, etc.

Once $\omega$ is chosen, assume that the admissible control function $v(t)$ in (5) is such that the function $\omega(|v|)$ is integrable. So, when $w(\xi)=\xi, v$ is a $\mathbb{L}_{1}$-function, when $w(\xi)=\xi^{2}, v$ is a $\mathbb{L}_{2}$-function, etc. Thus, the function $\omega(\xi)$ determines the class of admissible controls in (5).

Note that, by setting $v=\dot{x}$, all the above examples (1)-(4) fall within the formulation of (5), with $V=\mathbb{R}$.

To achieve the above goal, we exploit the extension approach of [13], based on generalized controls, by upgrading it to the case of discontinuous arcs. The case of continuous arcs, however, is still encompassed. Ultimately, the proposed extension links the approach developed by Gamkrelidze with the approaches in $[27,28,30]$, and by some other authors (see $[8,20,23]$ ). Moreover, it also generalizes the extensions from $[5,6,19]$, performed for control problems whose dynamics are linear in $v$, with separated control variables.

Overall, the line of investigation undertaken here is, of course, a road well traveled, and based on the so-called "graph completion" or "discontinuous time variable change" technique combined with the Gamkrelidze compactification, or convexification, technique. Besides the above-mentioned sources, our main line of research also goes along the works in [9,10,12,15-17,24,25,29,32]. This list, though, is far from being complete.

\section{Preliminaries}

To treat the extension, it is necessary to compactify the space $\mathbb{R}^{k}$ by adding a set $S_{\infty}^{k-1}$, called sphere at infinity. "Sphere at infinity" means that there is a homeomorphism $\Pi: S_{\infty}^{k-1} \rightarrow S^{k-1}$, where $S^{k-1}$ is the unit sphere in $\mathbb{R}^{k}$. The compactified space $\overline{\mathbb{R}}^{k}:=\mathbb{R}^{k} \cup S_{\infty}^{k-1}$ is endowed with a natural topology in which any sequence of points $v_{i} \in \mathbb{R}^{k}$ converges to the point $l \in S_{\infty}^{k-1}$ iff $\left|v_{i}\right| \rightarrow \infty$ and

$v_{i}=\left|v_{i}\right| \cdot \Pi(l)+o\left(\left|v_{i}\right|\right)$.

Note that the compact $\overline{\mathbb{R}}^{k}$ is topologically equivalent to the closed unit ball $B_{\mathbb{R}^{k}}$ in $\mathbb{R}^{k}$ due to the following homeomorphism $\Theta$, defined by

$\Theta(v)=\frac{v}{1+|v|}, \quad v \in \mathbb{R}^{k}$,

and $\Theta(v)=\Pi(v)$ if $v \in S_{\infty}^{k-1}$.

Denote

$V_{\infty}:=\Pi^{-1}\left(\operatorname{Limsup}_{|v| \rightarrow \infty} \frac{v}{|v|}\right)$.

Here $|v| \stackrel{V}{\rightarrow} \infty$ means that $|v| \rightarrow \infty$ and $v \in V$.

Now, the set $\bar{V}:=V \cup V_{\infty}$ is compact.

Let us introduce our main hypothesis about the right-hand side $g$ and function $g_{0}$. Assume that there exists a continuous function $g^{\infty}: \mathbb{R}^{n} \times S^{k-1} \times \mathbb{R}^{1} \rightarrow \mathbb{R}^{n}$,

such that

$\lim _{v \rightarrow \Pi^{-1}(e)} \frac{g(x, v, t)}{\omega(|v|)}=g^{\infty}(x, e, t) \quad \forall x, t \in \mathbb{R}^{n} \times \mathbb{R}^{1}, \quad \forall e \in S^{k-1}$.

Then, there is defined a continuous function $\bar{g}: \mathbb{R}^{n} \times \overline{\mathbb{R}}^{k} \times \mathbb{R}^{1} \rightarrow \mathbb{R}^{n}$ such that

$\bar{g}(x, v, t)=\frac{g(x, v, t)}{1+\omega(|v|)} \quad$ if $v \in \mathbb{R}^{k}$,

and $\bar{g}(x, v, t)=g^{\infty}(x, \Pi(v), t)$, if $v \in S_{\infty}^{k-1}$.

In a similar way, assume that there exist a function $g_{0}^{\infty}: \mathbb{R}^{n} \times S^{k-1} \times \mathbb{R}^{1} \rightarrow \mathbb{R}^{1}$, and a function $\bar{g}_{0}: \mathbb{R}^{n} \times \overline{\mathbb{R}}^{k} \times \mathbb{R}^{1} \rightarrow \mathbb{R}^{1}$ which are defined just as above but using $g_{0}$ instead of $g$.

We shall use the following assumptions.

(H1) Functions $\bar{g}$, and $\bar{g}_{0}$ introduced above do exist. Moreover, the function $\bar{g}$ is such that

$|\bar{g}(x, v, t)| \leq m(t) \kappa(|x|) \quad \forall(x, v, t) \in \mathbb{R}^{n} \times \bar{V} \times \mathbb{R}^{1}$,

where $\kappa: \mathbb{R}^{1} \rightarrow \mathbb{R}^{1}$ is such that

$\frac{\kappa(|x|)}{1+|x|} \leq$ const $\quad \forall x$,

and $m$ is some locally integrable function.

(H2) Functions $\bar{g}$, and $\bar{g}_{0}$ are continuously differentiable in $x, t$ for all $v \in \overline{\mathbb{R}}^{k}$.

Definition 1. The control problem (5) is said to allow the impulsive extension of order $\omega$ provided the hypothesis (H1) is satisfied and at least one of the functions $g^{\infty}$ or $g_{0}^{\infty}$ is not a zero function.

It is easy to see that the Euler and Dido problems (1), (2), and (3) allow extension of linear order $\xi$ whereas the problem (4) already requires quadratic growth $\omega(\xi)=\xi^{2}$.

Consider a scalar Borel measure $\mu: \mathcal{B}(T) \rightarrow[0,+\infty), T=\left[t_{0}, t_{1}\right]$. Here, $\mathcal{B}(T)$ stands for the $\sigma$-algebra of Borel subsets of $T$. 
Denote by $\mathcal{D}(t ; \mu)$ the Radon-Nikodym derivative

$\mathcal{D}(t ; \mu):=\frac{d \ell}{d(\ell+\mu)}$,

where $\ell$ is the Lebesgue measure on the real line.

Note that $\mathcal{D}(\cdot ; \mu)$ is $(\ell+\mu)$-measurable and takes values in $[0,1]$.

In what follows, a key role will be played by the so-called discontinuous time-variable change introduced first by $\mathrm{H}$. Lebesgue. This variable change is given by the function $\pi: T \rightarrow[0, c]$,

$\pi(t)=t-t_{0}+\mu\left(\left[t_{0}, t\right]\right), t \in\left(t_{0}, t_{1}\right], \quad \pi\left(t_{0}\right)=0$,

where $c=t_{1}-t_{0}+\|\mu\|$. Here $\|\mu\|=\mu\left(\left[t_{0}, t_{1}\right]\right)$ is the total variation.

It is a straightforward task to derive that there exists an inverse function $\theta(s):[0, c] \rightarrow T$ such that

(a) $\theta(s)$ is monotonically increasing;

(b) $\theta(s)$ is absolutely continuous and $|\theta(s)-\theta(t)| \leq|s-t| \forall s, t$;

(c) $\theta(s)=\tau \forall s \in \Gamma_{\tau}, \forall \tau \in T$, where $\Gamma_{\tau}=\left[\pi\left(\tau^{-}\right), \pi\left(\tau^{+}\right)\right]$.

Note that the function $\pi(t)$ maps $(\ell+\mu)$-measurable sets into $\ell$ measurable sets. Indeed, it follows directly from the definition of $\pi$ and from the representation of a set as a union of Borel and zero measure sets. Therefore, if the set $E$ is $(\ell+\mu)$-measurable, then $\theta^{-1}(E)$ is measurable. This implies that $v(\theta(s))$ is measurable provided that $v(t)$ is $(\ell+\mu)$-measurable. So, the variable change can be applied in the integral, i.e.,

$\int_{t_{0}}^{t_{1}} v(t) d \mu=\int_{0}^{c} v(\theta(s)) m(\theta(s)) d s$,

where $m(t)$ is the Radon-Nikodym derivative of measure $\mu$ w.r.t. $\ell+\mu$.

We designate the atomic set of $\mu$ by $\operatorname{Ds}(\mu)$ which is given by

$\operatorname{Ds}(\mu):=\{\tau \in T: \mu(\tau)>0\}$.

Recall that, due to [13], the generalized control is a weakly measurable family of probabilistic Radon measures $\nu_{t}$ : $\mathcal{B}(V) \rightarrow[0,1], t \in\left[t_{0}, t_{1}\right]$. "Weakly measurable" means that for any continuous scalar function $h(v, t)$, the function

$\left\langle h(v, t), \nu_{t}\right\rangle:=\int_{V} h(v, t) d \nu_{t}$

is measurable in $t$. Similarly, "weakly $\mu$-measurable" means that the above function is $\mu$-measurable.

If the set $V$ is compact, then the set of generalized controls is weakly sequentially compact, [13]. However, if the set $V$ is unbounded, this is not the case anymore. In what follows, we shall widely use generalized controls and their properties. The reader can find more details and information on generalized controls in $[1,2,31]$, and in the references therein.

\section{Extension concept}

The extension for problem (5) acquires the form

(P) Minimize $\int_{t_{0}}^{t_{1}}\left\langle\bar{g}_{0}(x, v, t), d c\right\rangle$

subject to $\quad d x=\langle\bar{g}(x, v, t), d c\rangle$,

$x\left(t_{0}\right) \in A, x\left(t_{1}\right) \in B$,

$\varphi(x, t) \leq 0$

$\mathfrak{c}=\left\{\mu, \nu_{t}, \nu_{s}^{\tau}\right\}, \quad \operatorname{supp}(\mathfrak{c}) \subseteq \bar{V}$

The above formulae and notations require clarification. Next, we give the necessary definitions.
The symbol $c$ designates generalized impulsive control. By definition, it consists of the following three components:

- $\mu: \mathcal{B}(T) \rightarrow[0,+\infty)$ which is a non-negative scalar Borel measure;

- $\nu_{t}: \mathcal{B}(\bar{V}) \rightarrow[0,1]$ which is a weakly $(\ell+\mu)$-measurable family of Radon probabilistic measures concentrated on $\bar{V}$, depending on $t \in\left[t_{0}, t_{1}\right]$, such that

$$
\mathcal{D}(t ; \mu)+\int_{\bar{V}} \frac{\omega(|v|)}{1+\omega(|v|)} \cdot d \nu_{t}=1 \quad \text { a.a. } t \quad \text { w.r.t. } \ell+\mu ;
$$

- $\nu_{s}^{\tau}: \mathcal{B}\left(V_{\infty}\right) \rightarrow[0,1]$ which is a family of Radon probabilistic measures concentrated on $V_{\infty}$, depending on $s \in[0,1]$, and on $\tau \in \operatorname{Ds}(\mu)$, weakly $\ell$-measurable in $s$ for each $\tau \in \operatorname{Ds}(\mu)$.

Above we identify the Borel measure $\mu$ with its unique Lebesgue-Stiltjes extension. In the sequel, " $\mu$-measurable" means measurability in the sense of its unique Lebesgue-Stieltjes extension.

Thus, the families of measures $\left\{\nu_{t}\right\}_{t \in T}$, and $\left\{\nu_{s}^{\tau}\right\}_{S \in[0,1]}$, for each $\tau \in \operatorname{Ds}(\mu)$, are generalized controls. The family of generalized controls $\left\{\nu_{s}^{\tau}\right\}_{\tau \in \mathrm{Ds}(\mu)}$ is called attached to the control measure $\mu$.

The symbolic notation $\operatorname{supp}(\mathfrak{c}) \subset \bar{V}$ refers to the above conditions imposed on the supports of $\nu_{t}$, and $\nu_{s}^{\tau}$.

Let us now proceed to the concept of trajectory. Denote by $x_{\tau}(s)$ the solution to the attached differential system

$\left\{\begin{array}{l}\dot{x}_{\tau}(s)=\Delta_{\tau} \cdot\left\langle\bar{g}\left(x_{\tau}(s), v, \tau\right), \nu_{s}^{\tau}\right\rangle, s \in[0,1] \\ x_{\tau}(0)=x\left(\tau^{-}\right)\end{array}\right.$

where $\Delta_{\tau}:=\mu(\{\tau\}), \tau \in \operatorname{Ds}(\mu)$.

The extended $\operatorname{arc} x(t),\left\{x_{\tau}(s)\right\}_{\tau \in \operatorname{Ds}(\mu)}$ is said to be a solution to the differential equation (8) corresponding to the initial value $x_{A} \in A$, if and only if,

$x(t)=x_{A}+\int_{t_{0}}^{t}\left\langle\bar{g}(x, v, \varsigma), \nu_{\varsigma}\right\rangle d\left(\ell+\mu_{c}\right)+\sum_{\tau \in \operatorname{Ds}(\mu): \tau \leq t}\left(x_{\tau}(1)-x_{\tau}(0)\right)$

for all $t \in\left(t_{0}, t_{1}\right]$, and $x\left(t_{0}\right)=x_{A}$.

By taking into account what has already been introduced, this definition clarifies the notion of integral in (7):

$$
\begin{aligned}
\int_{t_{0}}^{t_{1}}\left\langle\bar{g}_{0}(x, v, t), d c\right\rangle= & \int_{t_{0}}^{t_{1}}\left\langle\bar{g}_{0}(x, v, \varsigma), \nu_{\varsigma}\right\rangle d\left(\ell+\mu_{c}\right) \\
& +\sum_{\tau \in \operatorname{Ds}(\mu): \tau \leq t} \int_{0}^{1} \Delta_{\tau}\left\langle\bar{g}_{0}\left(x_{\tau}(s), v, \tau\right), \nu_{s}^{\tau}\right\rangle d s .
\end{aligned}
$$

It remains to give a meaning to the state constraints (10). Note that the state constraints $\varphi(x, t) \leq 0$ here should be understood in a wider sense than that of a conventional inequality. This is due to the presence of the attached family of trajectories $x_{\tau}(s)$.

Namely,

$\varphi(x, t) \leq 0 \Leftrightarrow \begin{cases}\varphi(x(t), t) \leq 0 & \forall t \in T, \\ \varphi\left(x_{\tau}(s), \tau\right) \leq 0 & \forall s \in[0,1], \forall \tau \in \operatorname{Ds}(\mu) .\end{cases}$

Problem (P) indeed represents an extension for problem (5) since for any control $v$ admissible to the problem (5) there is a control $c$ of the problem $(\mathrm{P})$ such that the corresponding trajectories $x(\cdot)$ and cost functional values coincide. Indeed, let $v(\cdot)$ be a control function for (5). Consider the absolutely continuous Borel measure

$\mu(C)=\int_{C} \omega(|v(t)|) d t, \quad C \in \mathcal{B}(T)$,

and take $\nu_{t}=\delta_{v(t)}$ a.a. $t \in T$, where $\delta_{r}$ designates Dirac's measure concentrated at point $r \in \mathbb{R}^{m}$. It is clear that

$\mathcal{D}(t ; \mu)=1 /(1+\omega(|v(t)|))$,

and, hence $\nu_{t}$ satisfies (11). 
Taking into account definition of $\bar{g}$ :

$$
\begin{aligned}
x(t) & =x_{A}+\int_{t_{0}}^{t}\langle\bar{g}(x, v, \varsigma), d \mathfrak{c}\rangle \\
& =x_{A}+\int_{t_{0}}^{t} \int_{v \in V}\left\langle\frac{g(x(\varsigma), v, \varsigma)}{1+\omega(|v(\varsigma)|)}, d \delta_{v(\varsigma)}\right\rangle(1+\omega(|v(\varsigma)|)) d \varsigma \\
& =x_{A}+\int_{t_{0}}^{t} g(x(\varsigma), v(\varsigma), \varsigma) d \varsigma .
\end{aligned}
$$

The pair $(x, c)$ is called control process if (8) holds true. A control process is said to be admissible if the endpoint constraints (9) and state constraints (10) are satisfied. Let us denote the set of all admissible processes by $\mathcal{P}$. An admissible process $\left(x^{*}, c^{*}\right)$ is said to be optimal or a solution to $(\mathrm{P})$ if the least possible finite value of the integral in (7) over all elements from $\mathcal{P}$ is reached by $\left(x^{*}, c^{*}\right)$.

The extension concept expressed by the problem (P) naturally generalizes the approaches in [13], [27], and [28], and also the recently constructed extension in [5] defined for the case of linear dependence in $v$. Note that all the examples (1)-(4) considered in the introduction possess extensions in the framework of the above proposed approach.

\section{Existence theorem}

The main goal of any extension undertaken in the optimal control theory is to ensure the existence of solution for the extended problem. The following theorem demonstrates consistency of the extension $(\mathrm{P})$ in the just mentioned sense.

Theorem 1. Suppose that the control problem (5) allows the impulsive extension of order $\omega$. Let either the set $A$ or the set $B$ be compact, $\mathcal{P} \neq \varnothing$, and assume that there exist constants $\kappa$, and $C>0$ such that, for any $(x, c) \in \mathcal{P}$, where $\mathfrak{c}=\left\{\mu, \nu_{t}, \nu_{s}^{\tau}\right\}$, at least one of the following conditions is satisfied:

(a) $\|\mu\| \leq \kappa \int_{t_{0}}^{t_{1}}\left\langle\bar{g}_{0}(x, v, t), d c\right\rangle+C$;

(b) $\|\mu\| \leq C$.

Then, the problem $(P)$ has a solution.

Proof. The main idea of the proof is to reduce the problem $(P)$ to a conventional convex control problem by using the discontinuous time variable change $\pi$ given by (6) and the "cosmic" variable change $\Theta$ (see Section 2).

The variable change $\Theta$, though, needs a slight upgrade. Let

$\Theta_{\omega}(v)=\frac{\omega(|v|) \cdot v}{|v|(1+\omega(|v|))}, \quad v \in \mathbb{R}^{k}$,

and $\Theta_{\omega}(v)=\Pi(v)$ when $v \in S_{\infty}^{k-1}$.

Consider the conventional optimal control problem with generalized controls

$$
\begin{array}{cl}
\text { Minimize } & \int_{0}^{s_{1}}\left\langle f_{0}(x, u, \chi), \xi_{s}\right\rangle d s, \\
\text { subject to } & \dot{x}=\left\langle f(x, u, \chi), \xi_{s}\right\rangle, \\
& \dot{\chi}=\alpha, \text { a.a. } s \in\left[0, s_{1}\right], \\
& x(0) \in A, \chi\left(s_{1}\right) \in B, \\
& \chi(0)=t_{0}, \chi\left(s_{1}\right)=t_{1}, \\
& \varphi(x, \chi) \leq 0, \\
& \alpha(s) \in[0,1], \\
& \alpha(s)+\left\langle|u|, \xi_{s}\right\rangle=1 \text { a.a. } s, \\
& \operatorname{supp}\left(\xi_{s}\right) \subseteq U \forall s \in\left[0, s_{1}\right] .
\end{array}
$$

Here, $f_{0}(\chi, u, \chi)=\bar{g}_{0}\left(\chi, \Theta_{\omega}^{-1}(u), \chi\right), f(x, u, \chi)=\bar{g}\left(\chi, \Theta_{\omega}^{-1}(u), \chi\right)$, and $U=\Theta_{\omega}(\bar{V})$.

Unlike in the problem $(\mathrm{P})$ which is considered on the fixed time interval $\left[t_{0}, t_{1}\right]$, the time $s_{1}$ in (12) is not fixed. The controls in (12) are $\alpha(s)$, and $\xi_{s}$, where $\alpha$ is the usual control (measurable function) and $\xi_{s}$ is the generalized control having support in $U$. Note that the set $U$ is compact since it is the image of the compact $\bar{V}$ by the continuous map $\Theta_{\omega}$.

Thus, the problem (12) is a conventional autonomous convex control problem with free time, see [13].

Next we show that the two problems, (P) and (12), are equivalent. That is, for every process $(x, c) \in \mathcal{P}$ there exists an admissible process $\left(\tilde{x}, \chi, \alpha, \xi_{s}, s_{1}\right)$ of the problem (12), such that the values of the minimizing integrals are equal, and vice versa.

Let $(x, \mathfrak{c}) \in \mathcal{P}$, where $\mathfrak{c}=\left\{\mu, \nu_{t}, \nu_{s}^{\tau}\right\}$. Consider the discontinuous time variable change $\pi(t)$. The inverse to $\pi$ function is $\theta(s)$ : $\left[0, s_{1}\right] \rightarrow T$, where we put $s_{1}=t_{1}-t_{0}+\|\mu\|$.

Let us take

$$
\begin{gathered}
\alpha(s)= \begin{cases}\mathcal{D}(\theta(s) ; \mu), & \text { if } s \in H(\mu), \\
0, & \text { otherwise, }\end{cases} \\
\xi_{s}= \begin{cases}\vartheta_{\theta(s)}, & \text { if } s \in H(\mu), \\
\vartheta_{\zeta_{\tau}(s)}^{\tau}, & \text { otherwise, }\end{cases} \\
\tilde{x}(s)= \begin{cases}x(\theta(s)), & \text { if } s \in H(\mu), \\
x_{\tau}\left(\zeta_{\tau}(s)\right), & \text { otherwise, }\end{cases}
\end{gathered}
$$

where $\vartheta_{t}$, and $\vartheta_{s}^{\tau}$ are generalized controls on $U$, such that

$$
\begin{array}{ll}
\vartheta_{t}(E)=\nu_{t}\left(\Theta_{\omega}^{-1}(E)\right) & \forall E \in \mathcal{B}(U), \\
\vartheta_{s}^{\tau}(E)=\nu_{s}^{\tau}\left(\Theta_{\omega}^{-1}(E)\right) & \forall E \in \mathcal{B}(U),
\end{array}
$$

and $H(\mu)$ is defined by

$H(\mu):=\left[0, s_{1}\right] \backslash \bigcup_{\tau \in \operatorname{Ds}(\mu)} \Gamma_{\tau}, \quad \zeta_{\tau}(s):=\frac{s-\pi\left(\tau^{-}\right)}{\ell\left(\Gamma_{\tau}\right)}: \Gamma_{\tau} \rightarrow[0,1]$.

Note that the definitions of $\vartheta_{t}$, and $\vartheta_{s, \tau}$ are correct since $\mathcal{B}(U)=$ $\Theta_{\omega}(\mathcal{B}(\bar{V}))$.

Notice that

$\theta(s)=t_{0}+\int_{0}^{s} \alpha(\varsigma) d \varsigma$

Indeed, by definition, we have

$\ell\left(\left[t_{0}, t\right]\right)=t-t_{0}=\int_{t_{0}}^{t} \mathcal{D}(\sigma ; \mu) d \pi(\sigma)=\int_{0}^{\pi(t)} \mathcal{D}(\theta(\varsigma) ; \mu) d \varsigma$.

From here, taking into account definitions of $\alpha, \theta$ and also the fact that $\pi(\theta(s))=s$ whenever $\pi(t)$ is continuous at the point $t=\theta(s)$, and by putting in the above $t=\theta(s)$, we obtain (13).

Thus, $\chi=\theta$.

It is easy to see that thanks to (11) the constraint $\alpha(s)+\left\langle|u|, \xi_{s}\right\rangle=1$ imposed in (12) is satisfied for a.a. $s$. It is also clear that the endpoint constraints and state constraints in (12) are satisfied.

By performing the variable change in (8) and by taking into account the trajectory concept, it follows that the arc $\tilde{x}(\cdot)$ satisfies the dynamical system in (12) together with the collection $\left(x_{A}, \alpha, \xi_{S}, s_{1}\right)$. Also due to the variable change (13), and to the definition of integral in (7), we have that

$\int_{t_{0}}^{t_{1}}\left\langle\bar{g}_{0}(x, v, t), d c\right\rangle=\int_{0}^{s_{1}}\left\langle f_{0}(\tilde{x}(s), u, \chi(s)), \xi_{s}\right\rangle d s$. 
So, the above constructed process $\left(\tilde{\chi}, \chi, \alpha, \xi_{s}, s_{1}\right)$ is admissible in (12). Moreover the cost function takes the same value.

Conversely, let us be given an admissible process $\left(\tilde{\chi}, \chi, \alpha, \xi_{s}, s_{1}\right)$ of the problem (12). Let us look at the function $\chi(s)$ as an inverse function to a certain discontinuous time variable change $\tilde{\pi}: T \rightarrow\left[0, s_{1}\right]$. The function $\tilde{\pi}(t)$ is defined uniquely as such a function satisfying $\tilde{\pi}(\chi(s))=s$, a.a. $s$ such that $\alpha(s)>0, \tilde{\pi}\left(t_{0}\right)=0$, $\tilde{\pi}\left(t_{1}\right)=s_{1}$, and $\tilde{\pi}(t)$ is right-continuous in $\left(t_{0}, t_{1}\right)$. Once $\tilde{\pi}$ is found, we obtain the measure $\mu$ satisfying

$\mu\left(\left[t_{0}, t\right]\right)=\int_{0}^{\tilde{\pi}(t)}(1-\alpha(s)) d s$.

Let us consider $\nu_{t}=\tilde{\xi}_{\tilde{\pi}(t)}$, and $\nu_{s}^{\tau}=\tilde{\xi}_{\tilde{\zeta}_{\tau}(s)}$, where

$\tilde{\xi}_{s}(E)=\xi_{s}\left(\Theta_{\omega}(E)\right), \quad \forall E \in \mathcal{B}(\bar{V})$,

$\tilde{\zeta}_{\tau}(S)=\ell\left(\tilde{\Gamma}_{\tau}\right) s+\tilde{\pi}\left(\tau^{-}\right):[0,1] \rightarrow \tilde{\Gamma}_{\tau}$,

$\tilde{\Gamma}_{\tau}=\left[\tilde{\pi}\left(\tau^{-}\right), \tilde{\pi}\left(\tau^{+}\right)\right], \quad \tau \in \operatorname{Ds}(\mu)$.

Now, the $\operatorname{arc} x(t)=\tilde{x}(\tilde{\pi}(t)), t \in T$, and $x_{\tau}(s)=\tilde{x}\left(\tilde{\zeta}_{\tau}(s)\right)$ is the solution to (8). It follows right away from the variable change that the endpoint and state constraints are satisfied. So, the constructed process $(x, \mathfrak{c})$, where $\mathfrak{c}=\left\{\mu, \nu_{t}, \nu_{s}^{\tau}\right\}$ is admissible for problem $(\mathrm{P})$, and the cost function takes on the same value.

Thus, we have shown that the problems (P) and (12) are equivalent. Moreover, for the established one-to-one correspondence it holds

$s_{1}=t_{1}-t_{0}+\|\mu\|$.

So, if a solution exists to one of the problems, then it also exists in the other problem. Let us show that the solution to the auxiliary problem (12) exists. Since $\mathcal{P} \neq \varnothing$, the set of admissible processes in problem (12) is not empty either. Consider a minimizing sequence $\left(x_{i}, \chi_{i}, \alpha_{i}, \xi_{s, i}, s_{1, i}\right)$. Since the set of generalized controls is weakly sequentially compact [13], there exists a generalized control $\xi$ such that, after passing to a subsequence, $\xi_{i} \stackrel{w}{\rightarrow} \xi$. Similarly, since the unit ball in $\mathbb{L}_{2}$ is weakly sequential compact, after passing to a subsequence, $\alpha_{i} \stackrel{w}{\rightarrow} \alpha$.

The notation $\stackrel{w}{\rightarrow}$ above stands for weak convergence of controls.

Whatever condition (a) or (b) of the theorem we consider, from (14), it follows that

$0<t_{1}-t_{0} \leq s_{1, i} \leq$ const.

By passing to a subsequence $s_{1, i} \rightarrow s_{1}>0, x_{A, i} \rightarrow x_{A}$ (or $x_{B, i} \rightarrow x_{B}$ ). The trajectory $(x, \chi)$ related to $\left(x_{A, i}, \alpha, \xi_{S}\right)$ exists on the whole time interval $\left[0, s_{1}\right]$ due to the conditions imposed in hypothesis (H1). It satisfies the endpoint and state constraints, since $\left(x_{i}, \chi_{i}\right) \rightrightarrows(\chi, \chi)$, see [13]. Moreover, it is easy to see that $\alpha(s)+\left\langle|u|, \xi_{s}\right\rangle=1$ a.a. $s$.

Due to the weak convergence,

$\int_{0}^{s_{1, i}}\left\langle f_{0}\left(x_{i}, u, \chi_{i}\right), \xi_{s, i}\right\rangle d s \rightarrow \int_{0}^{s_{1}}\left\langle f_{0}(x, u, \chi), \xi_{s}\right\rangle d s$.

Then the collection $\left(x, \chi, \alpha, \xi_{s}, s_{1}\right)$ represents a solution to (12) because the sequence $\left(x_{i}, \chi_{i}, \alpha_{i}, \xi_{s, i}, s_{1, i}\right)$ was minimizing. The latter implies existence of solution to the original problem (P).

The proof is complete.

Now, let us make an important note. Since any Borel measure can be weakly* approximated by absolutely continuous measures, and in view of the Gamkrelidze approximation lemma (see [13]), ${ }^{1}$ by using

${ }^{1}$ This lemma, in particular, says that any generalized control can be weakly approximated by conventional controls, that is, in the sense of weak convergence of generalized controls. the discontinuous time variable change (see the proof of the Theorem 1), and the definition of the generalized impulsive control $\mathfrak{c}$, it is easy to derive that for any $(x, \mathfrak{c}) \in \mathcal{P}$ there exists a sequence of control processes $\left(x_{i}, v_{i}\right)$ of the problem (5), not necessarily admissible in (5), such that

$\int_{t_{0}}^{t_{1}} \omega\left(\left|v_{i}\right|\right) d t \rightarrow\|\mu\|, \quad \int_{t_{0}}^{t_{1}} g_{0}\left(x_{i}, v_{i}, t\right) d t \rightarrow \int_{t_{0}}^{t_{1}}\left\langle\bar{g}_{0}(x, v, t), d c\right\rangle$,

$x_{i}(t) \rightarrow x(t) \quad \forall t \in(T \backslash \operatorname{Ds}(\mu)) \cup\left\{t_{0}\right\} \cup\left\{t_{1}\right\}, \quad$ and

$\limsup _{i \rightarrow \infty} \max _{t \in T} \varphi^{j}\left(x_{i}(t), t\right) \leq 0 \quad \forall j=1, \ldots, l$.

This means that the conditions (a), and (b) of the Theorem 1 will be satisfied as soon as there are positive constants $\varepsilon, \kappa$, and $C$, and a number $i$, such that

(a') $\int_{t_{0}}^{t_{1}} \omega(|v|) d t \leq \kappa \int_{t_{0}}^{t_{1}} g_{0}(x, v, t) d t+C$, or

(b') $A$, and $B$ are compact, and $\int_{t_{0}}^{t_{1}} \omega(|v|) d t \leq \kappa \int_{t_{0}}^{t_{1}} g^{i}(x, v, t) d t+C$,

where $v(t) \in V$, and $\dot{x}=g(x, v, t)$ are such that $x\left(t_{0}\right) \in A$, $\max _{t \in T} \varphi^{j}$ $(x(t), t) \leq \varepsilon \forall j$.

Let us go back to the examples given in the Introduction. By means of what has been proved, the extension $(\mathrm{P})$ is successful for the problems (1)-(4) in the sense that there exists a solution to the extended problem. Let us confirm it by using the just derived conditions $\left(a^{\prime}\right)$ and $\left(b^{\prime}\right)$.

Regarding Dido problem (2) and the Catenary problem (3), it is obvious, because by setting $\omega(\xi)=\xi, v=\dot{x}, g^{1}(x, v):=v$, and $g^{2}(x, v)=\sqrt{1+v^{2}}$, we have

$\int_{0}^{1}|v| d t \leq \int_{0}^{1} \sqrt{1+|v|^{2}} d t=\int_{0}^{1} g^{2}(x, v) d t$.

Then, the condition $\left(b^{\prime}\right)$ guarantees the existence of solution.

For problem (4), the condition ( $\left.\mathbf{b}^{\prime}\right)$ works again with $\omega(\xi)=\xi^{2}$, $g^{1}(x, v):=v$, and $g^{2}(x, v)=v^{2}$, and, thus, the solution exists.

Regarding the Euler example (1), the existence of solution is also obvious if an additional constraint $x(t) \geq \varepsilon$, where $\varepsilon>0$, is imposed. Indeed, this is due to condition $\left(a^{\prime}\right)$ and the estimate

$\varepsilon \int_{0}^{1}|v| d t \leq \int_{0}^{1} \varepsilon \sqrt{1+|v|^{2}} d t \leq \int_{0}^{1} x \sqrt{1+|\dot{x}|^{2}} d t$

However, the case $x(t) \geq 0$ requires additional considerations. It does not follow from Theorem 1 directly, though can be proved by writing out the solutions to $\varepsilon$-problems and by passing to limit as $\varepsilon \rightarrow 0$.

Thus, we have shown, that thanks to $\left(a^{\prime}\right)$ and $\left(b^{\prime}\right)$ and Theorem 1 , it becomes possible to a priori guarantee whether the extension $(\mathrm{P})$ is successful or not, by operating only in terms of the original problem (5) which is being extended.

\section{Model example}

The classic examples demonstrated in the beginning of the article are rather simple, though they point out at the theoretical necessity of a proper extension. Let us provide a more constructive and less trivial example which, at the same time, connects the current theory with applied engineering problems. Here, we improve the example from [5].

Consider a controllable disk which we need to steer while avoiding undesired rotations. The disk has four small jet-engines called thrusters as shown at Fig. 1. The angle of each thruster can be chosen to change the line of propulsion. Therefore, in order to avoid rotation, all the force lines by each thruster have to intersect the center of gravity (CG) at any moment in time (see Fig. 2). Assume now that the CG position is some function of mass $m$. Fuel 


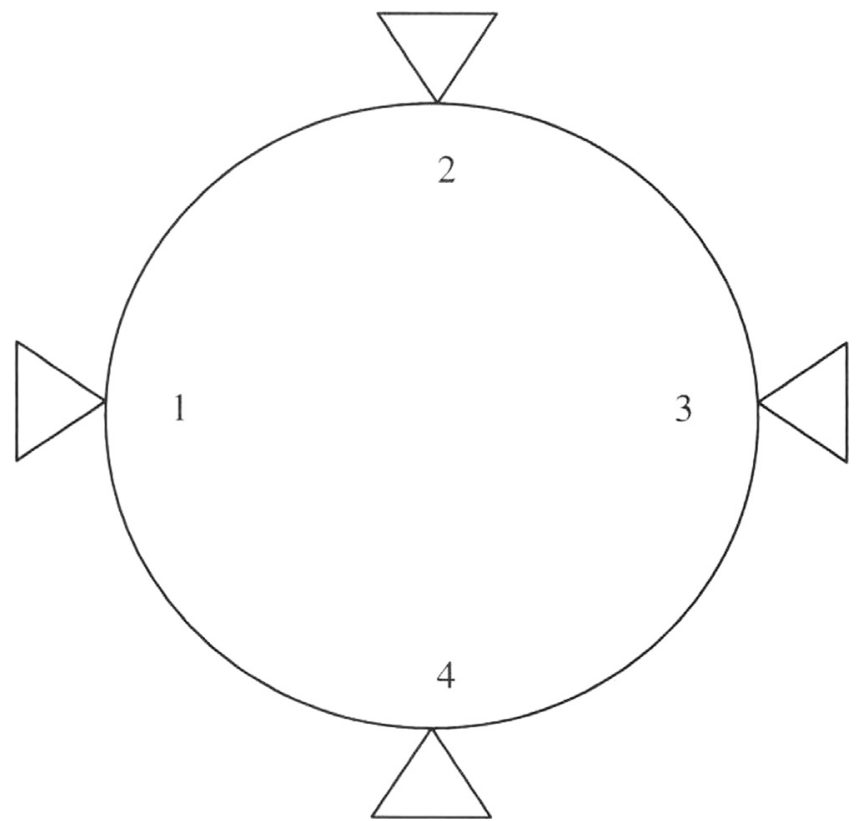

Fig. 1. Disk with four thrusters.

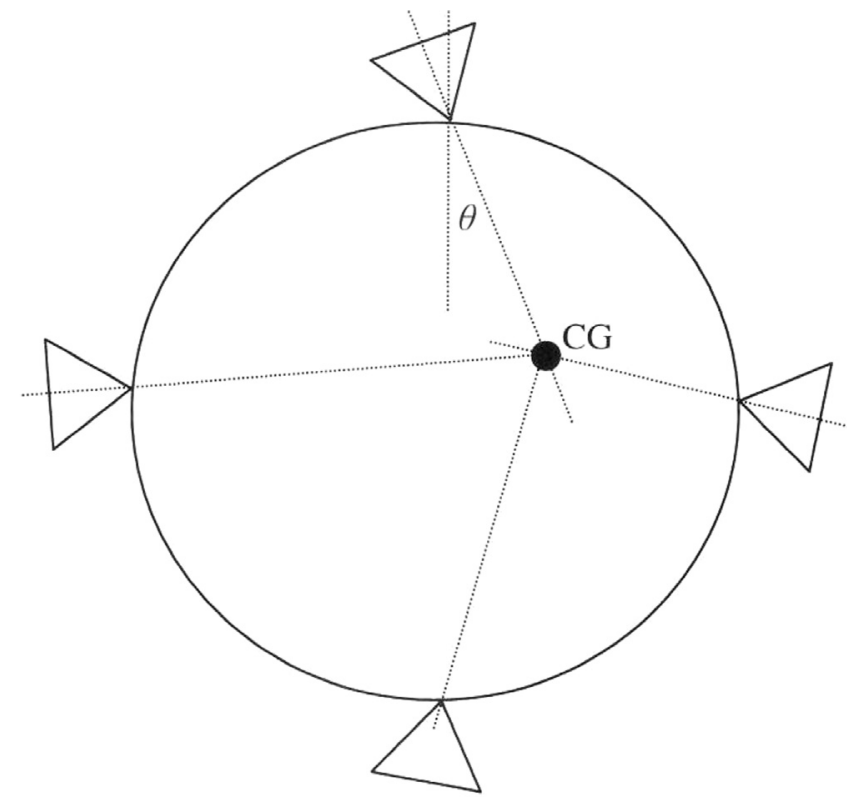

Fig. 2. Angle $\theta$ is restricted by the CG position.

consumption is related to the mass loss. Therefore, the thruster angles are also functions of mass, $\theta_{i}=\theta_{i}(m)$.

At each moment in time $t$, we control the thrusters relative output $p_{i}(t) \geq 0, i=1,2,3,4$ :

$p_{1}+p_{2}+p_{3}+p_{4}=1$,

and the fuel consumption rate $v=v(t) \geq 0$.

Assume that the control function $v(t)$ may take unbounded values. This is not really achievable in practice, but, in practice, the values of $v$ can be extremely large, thus justifying the above considered abstract mathematical model.

In what follows, the propulsion force is assumed to depend non-linearly on the fuel consumption. This is a quite realistic assumption. Therefore, we suppose that $F_{i}=p_{i} \phi(v)$, where $F_{i}$ is the propulsion force produced by the $i$-thruster, and $\phi(v)$ is a certain non-linear function characterizing fuel consumption at various engine modes. (Note that $\phi$ should be continuous.)

Regarding the function $\phi$, it is natural to assume that

$\lim _{v \rightarrow \infty} \frac{\phi(v)}{v}=\kappa>0$,

where $\kappa$ is an important characteristic of the jet-engine. For example, $\phi(v)=\kappa \sqrt{v^{2}-1}, v \geq 1$ and $\phi(v)=0$ when $v \in[0,1]$.

Let

$k_{1}(m, p)=p_{1} \cos \theta_{1}(m)-p_{2} \sin \theta_{2}(m)-p_{3} \cos \theta_{3}(m)+p_{4} \sin \theta_{4}(m)$,

$k_{2}(m, p)=-p_{1} \sin \theta_{1}(m)-p_{2} \cos \theta_{2}(m)+p_{3} \sin \theta_{3}(m)+p_{4} \cos \theta_{4}(m)$

Now, the motion of disc in the Earth's gravitational field is described by the equations:

$\dot{x}_{1}=w_{1}$,

$\dot{x}_{2}=w_{2}$,

$\dot{w}_{1}=\frac{k_{1}(m, p)}{m} \cdot \phi(v)$,

$\dot{w}_{2}=\frac{k_{2}(m, p)}{m} \cdot \phi(v)-g$,

$\dot{m}=-v$.

By fixing time interval $\left[t_{0}, t_{1}\right]$, the initial and terminal points $A$, and $B$, and, for example, the initial velocity at $A$, we obtain a boundary problem. By minimizing the functional

$m\left(t_{0}\right)-m\left(t_{1}\right)=\int_{t_{0}}^{t_{1}} v(t) d t$

on the solutions of the boundary problem, we obtain the so-called fuel minimization problem. Its solution fails to exist in the class of integrable controls and absolutely continuous trajectories. However, the solution can be found in the class of generalized impulsive controls and discontinuous arcs in view of the extension $(\mathrm{P})$, thanks to Theorem 1 (see the condition (a ') with $\omega(\xi)=\xi$ ). For this, we only need to ensure existence of at least one admissible arc.

The position and velocity of the disk may be restricted by some constraints. For example, the disk cannot fly under the ground. Thus, $x_{2} \geq 0$. It is also clear that the modulus of velocity $|w|$ should not exceed certain limits due to certain technological restrictions (air resistance, thermal barrier, etc.). Thus, $|w| \leq C$. So, in this way, the state constraints (10) naturally complement the problem formulation.

\section{Maximum principle}

In this section, we provide necessary conditions of optimality in the form of Pontryagin Maximum Principle for the extended problem (P).

Let us introduce necessary notations. Let

$\bar{H}: \mathbb{R}^{n} \times \overline{\mathbb{R}}^{k} \times \mathbb{R}^{n} \times \mathbb{R}^{l} \times \mathbb{R}^{1} \times \mathbb{R}^{1} \rightarrow \mathbb{R}^{1}$

be the extended Pontryagin function:

$\bar{H}\left(x, v, \psi, \gamma, t, \lambda^{0}\right):=\langle\psi, \bar{g}(x, v, t)\rangle-\langle\gamma, \Gamma(x, v, t)\rangle-\lambda^{0} \bar{g}_{0}(x, v, t)$,

where

$\Gamma(x, v, t):=\frac{\partial \varphi}{\partial x}(x, t) \bar{g}(x, v, t)+\frac{\partial \varphi}{\partial t}(x, t)$

is the derivative with respect to the control system (see chapter 6 in [26]).

Let $N_{C}(x)$ denote the limiting normal cone to the set $C$ at point $x$, in the sense of Mordukhovich [22].

The set of all generalized controls $\mathrm{c}=\left\{\mu, \nu_{t}, \nu_{S}^{\tau}\right\}$ defined on $\bar{V} \times T$ is designated by $\mathfrak{c}$. 
Theorem 2. Let $\left(x^{*}, \mathrm{c}^{*}\right)$, where $\mathrm{c}^{*}=\left(\mu^{*}, \nu_{t}^{*}, \nu_{s}^{\tau *}\right)$, be an optimal process to problem (P). Assume that the hypotheses (H1), and (H2) are in force.

Then, there exist a number $\lambda^{0} \geq 0$, a vector function of bounded variation $\psi$, together with its attached family $\psi_{\tau}$, and a monotonically decreasing function $\gamma$, together with its attached family $\gamma_{\tau}$, where $\tau \in \operatorname{Ds}\left(\mu^{*}\right)$, such that:

C1 The pair $\left(x^{*}, \psi\right)$ satisfies the generalized Hamiltonian system:

$$
\left\{\begin{array}{l}
d x^{*}=\left\langle\frac{\partial \bar{H}}{\partial \psi}\left(x^{*}, v, \psi, \gamma, t, \lambda^{0}\right), d c^{*}\right\rangle \\
d \psi=-\left\langle\frac{\partial \bar{H}}{\partial x}\left(x^{*}, v, \psi, \gamma, t, \lambda^{0}\right), d c^{*}\right\rangle,
\end{array}\right.
$$

on the time interval $\left[t_{0}, t_{1}\right]$, whereas for the attached families $x_{\tau}^{*}, \psi_{\tau}, \tau \in \operatorname{Ds}\left(\mu^{*}\right)$, on $[0,1]$, it takes place the differential equations

$$
\left\{\begin{array}{l}
\dot{x}_{\tau}^{*}=\Delta_{\tau} \cdot\left\langle\frac{\partial \bar{H}}{\partial \psi}\left(x_{\tau}^{*}, \nu, \psi_{\tau}, \gamma_{\tau}, \tau, \lambda^{0}\right), \nu_{s}^{\tau *}\right\rangle \\
\dot{\psi}_{\tau}=-\Delta_{\tau} \cdot\left\langle\frac{\partial \bar{H}}{\partial x}\left(x_{\tau}^{*}, v, \psi_{\tau}, \gamma_{\tau}, \tau, \lambda^{0}\right), \nu_{s}^{\tau *}\right\rangle,
\end{array}\right.
$$

are satisfied on $[0,1]$ with, $x_{\tau}(0)=x\left(\tau^{-}\right)$, and $\psi_{\tau}(0)=\psi\left(\tau^{-}\right)$;

C2 The transversality conditions

$$
\begin{aligned}
& \psi\left(t_{0}\right)-\frac{\partial \varphi}{\partial x}\left(x_{0}^{*}, t_{0}\right) \gamma\left(t_{0}\right) \in N_{A}\left(x_{0}^{*}\right), \\
& \psi\left(t_{1}\right)-\frac{\partial \varphi}{\partial x}\left(x_{1}^{*}, t_{1}\right) \gamma\left(t_{1}\right) \in-N_{B}\left(x_{1}^{*}\right),
\end{aligned}
$$

where $x_{0}^{*}=x^{*}\left(t_{0}\right)$, and $x_{1}^{*}=x^{*}\left(t_{1}\right)$;

C3 The maximum condition

$$
\max _{\mathfrak{c} \in \mathbb{C}:\|\mu\|=\left\|\mu^{*}\right\|} \int_{t_{0}}^{t_{1}}\left\langle\bar{H}\left(v, t, \lambda^{0}\right), d \mathfrak{c}\right\rangle=\int_{t_{0}}^{t_{1}}\left\langle\bar{H}\left(v, t, \lambda^{0}\right), d c^{*}\right\rangle ;
$$

C4 The non-triviality condition

$$
\lambda^{0}+\left|\psi\left(t_{0}\right)\right|+\left|\gamma\left(t_{0}\right)\right|>0 .
$$

Moreover, $\quad \gamma(t)$, and $\gamma_{\tau}(s), \quad \tau \in \operatorname{Ds}\left(\mu^{*}\right), \quad$ satisfy the following conditions:

(a) Functions $\gamma^{j}, \gamma_{\tau}^{j}, j=1, \ldots, l$, are constant on any segment in which the optimal arc $x^{*}(t)$, and $x_{\tau}^{*}(s)$ belongs to the interior of the set generated by the $j$ th state constraint.

(b) Functions $\gamma^{j}$ are left continuous on the interval $\left(t_{0}, t_{1}\right)$, and $\gamma_{\tau}^{j}$ are left continuous on the interval $(0,1)$.

(c) Functions $\gamma^{j}$ are decreasing, with $\gamma^{j}\left(t_{1}\right)=0$, and $\gamma_{\tau}^{j}$ are decreasing, with $\gamma_{\tau}(0)=\gamma\left(\tau^{-}\right)$, and $\gamma_{\tau}(1)=\gamma\left(\tau^{+}\right)$.

Above, for brevity: in (C1), the dependence of the functions on $t$, and $s$ in the functions is omitted, in (C3), the values of $x^{*}(t), \psi(t)$, and $\gamma(t)$ replace the omitted arguments. The solutions to (C1) and the meaning of the integral in (C3) are understood in the sense defined before, via the attached families (see Section 3).

Proof. Let us provide a sketch of the proof. The full proof contains rather cumbersome calculations albeit straightforward. Note that the above Maximum Principle (MP) degenerates. That is, it can be satisfied by a trivial Lagrange multiplier as soon as one of the endpoints $x_{0}^{*}, x_{1}^{*}$ belongs to the boundary of the state constraint set (for the phenomenon of degeneracy see [3] and [4]). Therefore, in what follows, it is not restrictive to assume that $\varphi^{j}\left(x_{l}^{*}\right)<0 \forall j, l$.

As we saw in the proof of Theorem 1, problem (P) turns out to be equivalent to problem (12). Problem (12) is a conventional problem in generalized controls and with state constraints. Then, it becomes possible to apply the MP obtained in [1] to the optimal process of problem (12). Let $\left(\lambda^{0}, \tilde{\psi}, \eta\right)$ be the Lagrange multipliers associated with the optimal process of problem (12) by means of this MP. Here, $\eta$ is the multiplier related to the state constraints. It is a Borel measure due to the MP in [1] obtained along the approach of [11]. To proceed to the MP in the form provided in [14], the following variable change is needed:

$\gamma(t)=\int_{\left[t, t_{1}\right]} d \eta, \quad \psi(t):=\tilde{\psi}(t)+\gamma(t) \frac{\partial \varphi}{\partial x}(t)$.

(For details, see [7].)

The next step is to apply the discontinuous time variable change to the derived conditions and, as a consequence, obtain the conditions (C1)-(C4). This part of the argument is carried out in a standard way (see the proof of Theorem 1 in [5]). The proof is complete. $\quad \square$

Let us now describe the extension and also the extended solutions to the minimal surface problem (1). Regarding classical continuous solutions - they are already known - the optimal arc is the hyperbolic cosine, $x(t)=\operatorname{ch}(t-1 / 2)$, being $r_{1}=r_{2}=$ $\left(e^{-1 / 2}+e^{1 / 2}\right) / 2$. If the numbers $r_{1}$, and $r_{2}$ are sufficiently small, the discontinuous solution is obvious. Indeed, as it was mentioned in Introduction, it is given by $x(0)=r_{1}, x(1)=r_{2}$ and $x(t)=0$ if $t \in(0,1)$. However, according to the approach proposed here, the discontinuous solution is not only the discontinuous trajectory $x(t)$ itself, but also the attached family of the continuous $\operatorname{arcs} x_{\tau}(s)$, where $s \in[0,1]$, specifying the behavior of the trajectory along the jumps at points $\tau$.

Thereby, let us give the precise extension following $(\mathrm{P})$.

By definition, we have

$\bar{g}(x, v)=\frac{v}{1+|v|}, \quad \bar{g}_{0}(x, v)=x \frac{\sqrt{1+v^{2}}}{1+|v|}$,

and $\bar{g}(x, v)= \pm 1, \bar{g}_{0}(x, v)=x$, if $v \in S_{\infty}^{0}$ (i.e., if $\left.v= \pm \infty\right)$. Then, the optimal generalized impulsive control $c$ is:

$\mu=r_{1} \delta_{0}+r_{2} \delta_{1}, \quad \nu_{t}=0, \quad \nu_{s}^{0}=\delta_{-\infty}, \quad \nu_{s}^{1}=\delta_{+\infty}$.

The optimal trajectory $x(t)$ is

$x(t)= \begin{cases}r_{1}, & t=0, \\ 0, & t \in(0,1), \quad x_{0}(s)=r_{1}-r_{1} s, \quad x_{1}(s)=r_{2} s . \\ r_{2}, & t=1 .\end{cases}$

The form of this solution follows from its geometrical meaning and the principle of optimality (which says "a piece of optimal arc is again optimal"). The attached functions $x_{0}(s)$, and $x_{1}(s)$ demonstrate the linear character of the rupture at the endpoints. On the other hand, it is a straightforward exercise to make sure that this solution satisfies the maximum principle stated in Theorem 2. Indeed, we have

$\bar{H}\left(x, v, \psi, \gamma, \lambda^{0}\right)=(\psi+\gamma) \frac{v}{1+|v|}-\lambda^{0} x \frac{\sqrt{1+v^{2}}}{1+|v|}$,

and therefore, $d \psi=\lambda^{0}\left\langle\sqrt{1+v^{2}} /(1+|v|), d c\right\rangle$. It is easy to check that the following multipliers satisfy the Maximum Principle at the above-mentioned control process:

$\lambda^{0}=1, \quad \psi(t)= \begin{cases}-r_{1}, & t=0, \\ 0, & t \in(0,1), \\ r_{2}, & t=1,\end{cases}$

while $\psi_{0}(s)=r_{1} s-r_{1}, \psi_{1}(s)=r_{2} s, \gamma(t)=0 \forall t \in[0,1], \gamma_{0}(s)=0$, and $\gamma_{1}(s)=0$. This is due to the fact that the function

$\frac{v-\sqrt{1+v^{2}}}{1+v}$

is monotonically increasing as $v \geq 0$ (we skip the details). 
Regarding this extension of (1), it is also important to note the following. First notice that the value of the integral in (1) is not exactly the area of the minimal surface. To be so the value of the integral in (1) needs to be multiplied by $2 \pi$.

Let us compute the value of this integral at the discontinuous solution pointed out above. By definition, we have:

$$
\begin{aligned}
2 \pi \int_{0}^{1} \bar{g}_{0}(x, v) d c & =2 \pi \int_{0}^{1} r_{1} x_{0}(s) d s+2 \pi \int_{0}^{1} r_{2} x_{1}(s) d s \\
& =2 \pi \int_{0}^{1} r_{1}^{2}(1-s) d s+2 \pi \int_{0}^{1} r_{2}^{2} s d s=\pi r_{1}^{2}+\pi r_{2}^{2} .
\end{aligned}
$$

Thus, the answer is the area of two circles with radiuses $r_{1}$, and $r_{2}$. This is fully consistent with the physical interpretation of the problem. Indeed, the minimal surface in the discontinuous case consists of the two disks facing each other plus the segment $[0,1]$. The area of the segment is zero, and the area of the disks amounts exactly to the above value $\pi r_{1}^{2}+\pi r_{2}^{2}$.

\section{Conclusions}

Some optimal control problems do not have solution in the class of classical controls. This suggests the need of a relaxation or extension of the control problem ensuring the existence of a solution in some enlarged class of controls. In this article, we developed an extension for the case in which the control dynamics are given by

$\dot{x}(t)=g(x(t), v(t), t)$,

where the control function $v(\cdot)$ takes its values in some closed set $V$, not necessarily bounded. The optimization problem is to minimize an integral cost functional over arcs satisfying this equation, where we seek solutions in the extended sense enabling $x(\cdot)$ to have jumps. To achieve this goal, we exploit the approach of R.V. Gamkrelidze based on the generalized controls. The proposed extension, however, allows for discontinuous arcs, what leads to the notion of generalized impulsive control. The fact that $V$ is not compact requires a certain compactification of the space to ensure weak compactness of the generalized impulsive controls. In turn, this is crucial for the existence of solutions. As a result, a theorem for the existence of solution is obtained. A maximum principle for constrained impulsive control problems is proved. Thus, the state constraints are addressed in the impulsive control context.

Besides, a number of classical examples, such as the "minimal surface problem", "Dido problem", "catenary" and others find proper extensions in the framework of the approach proposed in this work.

\section{Acknowledgments}

The first and fourth authors were supported by CNPq (Brazil) “Sem Fronteiras" grant number 401689/2012-3. The first author was also supported by the Russian Foundation for Basic Research, grant number 13-01-00494. The third author was supported by FCT (Portugal) grant Incentivo/EEI/UI0147/2014, and FCT funded project PEst-OE/EEI/UI0147/2014. The second and fourth authors were supported through grant 2013/07375-0, São Paulo Research Foundation (FAPESP - CEPID), and CNPq (Brazil) grant number 479109/2013-3. The fourth author was also supported by CNPq (Brazil) grant number 309335/2012-4.

The authors are also grateful to the anonymous reviewers for their constructive feedback and insightful suggestions which greatly helped improve this article.

\section{References}

[1] A.V. Arutyunov, Perturbations of extremal problems with constraints, and necessary conditions for optimality, in: Mathematical Analysis, vol. 27 (in Russian). Akad. Nauk SSSR Vsesoyuz. Inst. Nauchn. i Tekhn. Inform., Moscow. Itogi Nauki i Tekhniki, 237 (1989) 147-235. Translated in J. Soviet Math. 54 (6) (1991) 1342-1400.

[2] A.V. Arutyunov, Extensions and perturbations of optimal control problems, Tr. Mat. Inst. Steklova 220 (1998) 27-34.

[3] A.V. Arutyunov, Optimality Conditions: Abnormal and Degenerate Problems, Kluwer Academic Publishers, Dordrecht, 2000.

[4] A.V. Arutyunov, S.M. Aseev, State constraints in optimal control. The degeneracy phenomenon, Syst. Control Lett. 26 (1995) 267-273.

[5] A.V. Arutyunov, D. Karamzin, F.L. Pereira, On a generalization of the impulsive control concept: controlling system jumps, Discrete Contin. Dyn. Syst. 29 (2011) 403-415.

[6] A.V. Arutyunov, D.Y. Karamzin, F. Pereira, Pontryagin's maximum principle for constrained impulsive control problems, Nonlinear Anal. 75 (2012) 1045-1057.

[7] A.V. Arutyunov, D.Y. Karamzin, F.L. Pereira, The maximum principle for optimal control problems with state constraints by R.V. Gamkrelidze: revisited, J. Optim. Theory Appl. 149 (2011) 474-493.

[8] A. Bressan, F. Rampazzo, On differential systems with vector-valued impulsive controls, Boll. Un. Mat. Ital. B 2 (7) (1988) 641-656.

[9] W.J. Code, P.D. Loewen, Optimal control of non-convex measure-driven differential inclusions, Set-Valued Var. Anal. 19 (2011) 203-235.

[10] W.J. Code, G.N. Silva, Closed loop stability of measure-driven impulsive control systems, J. Dyn. Control Syst. 16 (2010) 1-21.

[11] A.J. Dubovickil̆, A.A. Miljutin, Extremal problems with constraints, Ž. Vyčisl. Mat. i Mat. Fiz. 5 (1965) 395-453.

[12] N. Forcadel, Z. Rao, H. Zidani, State-constrained optimal control problems of impulsive differential equations, Appl. Math. Optim. 68 (2013) 1-19.

[13] R. Gamkrelidze, Principles of Optimal Control Theory, Tbilisi University Press, Tbilisi, 1977 (in Russian). Translation in Plenum Press, New York, 1978.

[14] R.V. Gamkrelidze, Optimal control processes for bounded phase coordinates, Izv. Akad. Nauk SSSR. Ser. Mat. 24 (1960) 315-356.

[15] E. Goncharova, M. Staritsyn, Optimization of measure-driven hybrid systems, J. Optim. Theory Appl. 153 (2012) 139-156.

[16] M. Guerra, A. Sarychev, Existence and Lipschitzian regularity for relaxed minimizers, in: Mathematical Control Theory and Finance, Springer, Berlin, 2008, pp. 231-250. http://dx.doi.org/10.1007/978-3-540-69532-5_13.

[17] M. Guerra, A. Sarychev, Frechet generalized trajectories and minimizers for variational problems of low coercivity, J. Dyn. Control Syst. (2014) 1-27.

[18] D. Hilbert, Mathematical Problems, Bulletin of the American Mathematical Society, 1902.

[19] D.Y. Karamzin, Necessary conditions for the minimum in an impulsive optimal control problem, J. Math. Sci. 139 (2006) 7087-7150.

[20] B.M. Miller, The generalized solutions of nonlinear optimization problems with impulse control, SIAM J. Control Optim. 34 (1996) 1420-1440.

[21] B. Mordukhovich, Existence of optimal controls, Itogi Nauki Tech. Sovr. Prob. Mat. 6 (1976) 207-261, in Russian; English transl. in J. Soviet Math. 7 (1977) $850-886$.

[22] B.S. Mordukhovich, Variational Analysis and Generalized Differentiation, Springer-Verlag, Berlin, 2005.

[23] M. Motta, F. Rampazzo, Nonlinear systems with unbounded controls and state constraints: a problem of proper extension, NoDEA Nonlinear Differ. Equ. Appl. 3 (1996) 191-216.

[24] F.L. Pereira, G.N. Silva, Necessary conditions of optimality for vector-valued impulsive control problems, Syst. Control Lett. 40 (2000) 205-215.

[25] F.L. Pereira, G.N. Silva, V. de Oliveira, Invariance for impulsive control systems, Avtomat. i Telemekh. (2008) 57-71. http://dx.doi.org/10.1134/S0005117908050068.

[26] L.S. Pontryagin, V.G. Boltyanskiü, R.V. Gamkrelidze, E.F., Mishchenko, Mathematical Theory of Optimal Processes, fourth ed., Nauka, Moscow, 1983.

[27] R.W. Rishel, An extended Pontryagin principle for control systems whose control laws contain measures, J. Soc. Ind. Appl. Math. Ser. A Control 3 (1965) 191-205.

[28] R.T. Rockafellar, Dual problems of Lagrange for arcs of bounded variation, in: Calculus of Variations and Control Theory, Proceedings of Symposium, Math. Res. Center, Univ. Wisconsin, Madison, WI, 1975; Dedicated to Laurence Chisholm Young on the occasion of his 70th birthday), Academic Press, New York, pp. 155-192, Publ. Math. Res. Center Univ. Wisconsin, No. 36.

[29] G.N. Silva, R.B. Vinter, Necessary conditions for optimal impulsive control problems, SIAM J. Control Optim. 35 (1997) 1829-1846.

[30] J. Warga, Variational problems with unbounded controls, J. Soc. Ind. Appl. Math. Ser. A Control 3 (1965) 424-438.

[31] J. Warga, Optimal Control of Differential and Functional Equations, Academic Press, New York, London, 1972.

[32] P.R. Wolenski, S. Žabić, A sampling method and approximation results for impulsive systems, SIAM J. Control Optim. 46 (2007) 983-998 (electronic). 